\title{
Single-polarity high-voltage amplifier to drive coarse-approach slip-stick piezoelectric motors
}

\author{
Cristian H. Belussi, Mariano Gómez Berisso, and Yanina Fasano ${ }^{a}$ \\ Instituto Balseiro and Low Temperatures Laboratory, \\ Centro Atómico Bariloche,8400 Bariloche, Argentina.
}

(Dated: December 29, 2012)

\begin{abstract}
Scanning probe microscopies rely on a coarse piezoelectric motor that approaches the measuring probe towards the studied sample in milimeter-range distances. These motors typically work in a slip-stick mode by exciting the piezos with sawtooth signals of hundreds of $\mathrm{V}$ and up to some $\mathrm{kHz}$. In this paper we introduce a single-polarity high-voltage amplifier based on discrete MOSFETtechnology components easy to purchase worldwide. The amplifier has an output signal up to $600 \mathrm{~V}, 100 \mathrm{~mA}$ output current, and a noise level below $2 \mu \mathrm{V} / \sqrt{\mathrm{Hz}}$. When loaded with the typical impedances of piezoelectric motors it has a high-voltage bandwidth of $4 \mathrm{kHz}$. In the low-input ranges, for $V_{\text {out }}=100 \mathrm{~V}$ the amplifier presents a slew-rate of $2 \mathrm{~V} / \mu \mathrm{s}$, and rise and fall times of $80 \mu \mathrm{s}$. The circuit was successfully used to drive the coarse piezoelectric motor of a home-made scanning tunnelling microscope.
\end{abstract}

\section{INTRODUCTION}

Tailoring the physical properties of nano-scale devices based on novel materials interesting for applications requires access to local electronic and magnetic properties at different temperatures. This problem can be investigated in research laboratories by means of scanning probe microscopies such as tunnelling [1], atomic [2] and magnetic force [3], and near-field [4]. These techniques allow the characterization of the physical properties of metals, semiconductors and superconductors with atomic resolution and in an extended range of temperatures. Nowadays, most of scanning probe microscopes base their scan method in coarse and fine piezoelectric motors. The performance and reliability of the coarse positioning system is particularly critical when cooling the microscopes down to low temperatures.

Typical solutions for the problem of approaching the microscope sensor to the sample down to millimeter distances are inchworm [5] and slip-stick [6-8] piezoelectric motors. During the last years, the latter ones have been most widely used. They profit from the reverse piezoelectric effect, namely the contraction or expansion of a material resulting from the application of an electric field. Slip-stick motors are based on piezoelectric legs that are put in contact with the mobile sensor-holder and driven by asymmetric signals, typically sawtooth [6] or cycloid-like [9]. During the slow part of the driving signal the sensor-holder remains stuck to the piezoelectric legs since the applied force does not exceed the static friction. During the fast part, this friction is overcome and the sensor-holder slides in a motion governed by the dynamic-friction coefficient. A net displacement of the sensor-holder towards the sample in millimeters range can be achieved by suitably choosing the asymmetry, amplitude and frequency of the driving signal.

A reliable actuation of such motors requires low-noise and high-voltage amplifiers, with output signal amplitudes in the range of 200-600 V. In order to properly follow the driving signal these amplifiers must have a broad bandwidth which is related to having high slew-rate and short rise and fall times. In addition, providing a high output current is crucial since slip-stick piezoelectric motors present a merely capacitive impedance, provided they are driven at frequencies off-resonance. Typical impedances for the equivalent circuit are in the range of $10-40 \mathrm{nF}$ providing the piezoelectric legs can be made by stacking several piezoelectric crystals in order to enhance the strain-to-driving voltage ratio [10]. Therefore, in order to produce one step with the motor, the driving electronics has to charge and discharge this capacitance with hundreds of Volts in microsecond times. The high-voltage amplifier required for this application has to posses two basic properties that are not trivial to meet at the same time, high output currents and high slew-rate.

Many commercial electronics to drive piezoelectric motors are available in the market though most of them are quite expensive $[11,12]$. In this paper we introduce an inexpensive single-polarity high-voltage amplifier for the discussed application. As shown in Sec. II, the amplifier circuit is based in discrete MOSFET-technology components that are easily purchased in most local markets. Section III presents experimental data on the main figures of merits of the amplifier when loaded with a $30 \mathrm{nF}$ capacitor: $4 \mathrm{kHz}$ bandwidth, $100 \mathrm{~mA}$ output current, $2 \mathrm{~V} / \mu$ s slew-rate, $80 \mu$ s rise and fall times for an output signal of $100 \mathrm{~V}$, and a noise level below $2 \mu \mathrm{V} / \sqrt{\mathrm{Hz}}$. Finally, Sec. IV provides a brief comparison with other high-voltage amplifiers proposed for driving piezoelectric devices and focus on the advantages of the one introduced here in order to drive slip-stick motors. 


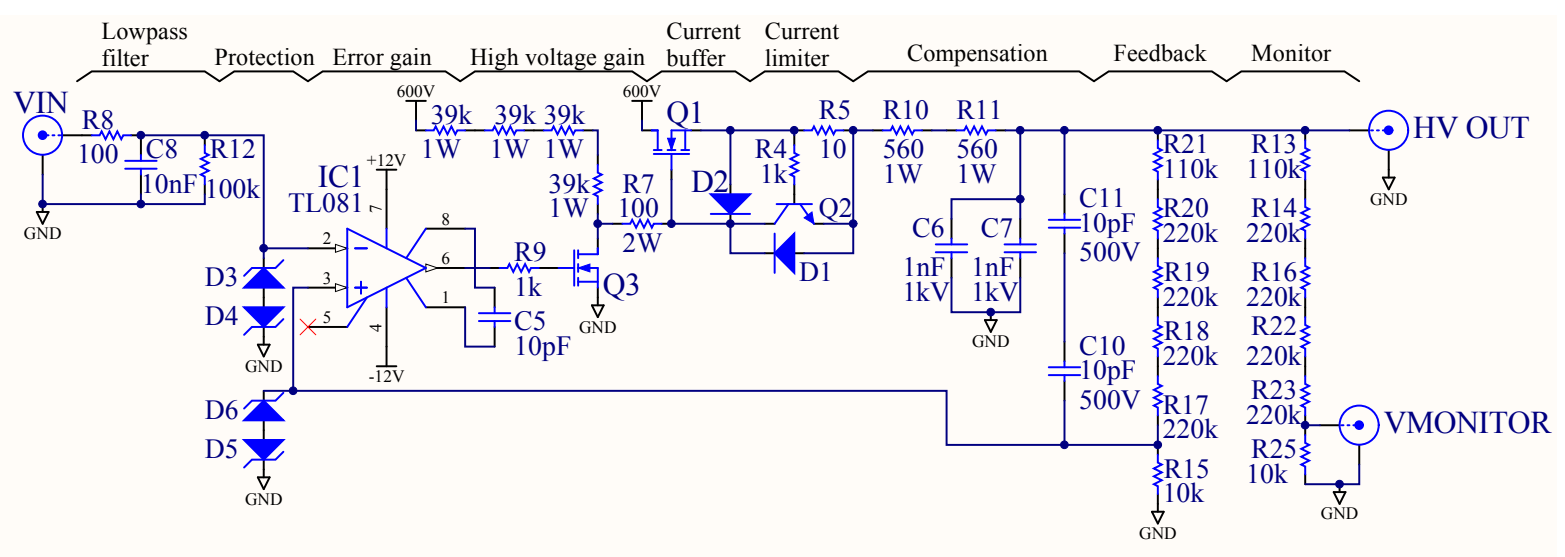

Figure 1: Detailed circuit for the up to $600 \mathrm{~V}$ high-voltage amplifier. The MOSFET transistors are Q1, Q3:BUZ80A; Q2, Q4, Q5:BC548C. The diodes are D1, D2:UF4007; D3, D4, D5, D6:DZ 12V. Resistances are given in Ohms and are of 0.5 W except indicated otherwise. Capacitances are given in Farads and have a voltage limit of $50 \mathrm{~V}$, except $\mathrm{C} 10$ and $\mathrm{C} 11$ with $500 \mathrm{~V}$ and C6 and $\mathrm{C} 7$ with $1 \mathrm{kV}$.

\section{HIGH-VOLTAGE AMPLIFIER CIRCUIT}

High-voltage amplifiers face important limitations when applied to drive piezoelectric motors. Notably, the loaddependent bandwidth of an amplifier is significantly reduced when driving a highly-capacitive circuit as that of piezoelectric stacks. This type of load, in combination with the amplifier finite output impedance, also produces phase-lag in the amplifier feedback path. In addition, at high-frequencies the current limit required for this load entails impractically high power-dissipation. For a more detailed discussion on this issue we refer the reader to Ref. 13. These reasons make difficult to develop a high-voltage amplifier for typical piezoelectric-motor loads with bandwidths exceeding $10 \mathrm{kHz}$.

Having this in mind, we focused our efforts in developing a discrete amplifier circuit with some $\mathrm{kHz}$ bandwidth but improved output current, slew-rate, and rise and fall times. The amplifier presented here is based in MOSFETtechnology components and has therefore a much lower cost than those based in integrated high-voltage operational amplifiers [20]. One important characteristic of our amplifier is its single-polarity that allows to avoid the undesirable reversal of polarization that can occur when applying significant negative voltages.

The amplifier has a gain of 100 and a maximum output voltage of $600 \mathrm{~V}$. The main technical data of the circuit are shown in Table I. Figure 1 shows a detailed diagram of the amplifier circuit that basically consists in three stages. In the first one, a TL081 operational amplifier with relatively high input impedance linearizes the circuit. The second stage consists on a high-voltage n-MOSFET transistor (Q3 in Fig. 1) and its associated components. This configuration works as a common source, provides high-voltage gain, and drives the gate of the transistor of the following stage. This third stage has the same n-MOSFET transistor (Q1 in Fig. 1) that works both, as a common drain when the input signal increases, and as a push-pull circuit when it decreases. This performance is due to the presence of the diode D2 connected between the gate and the source of Q1. The remaining components of the circuit provide the feedback path (R15, R17, R18, R19, R20, and R21), protection (D6, D5, D3, D4, and Q2), and compensation (R10, R11, C10, C11, and C5). The feedback path resistances limit the gain of the amplifier at low frequencies. The circuit includes an auxiliary low-voltage output in order to monitor the high-voltage output.

Since special attention was put on providing a high output current, Q1 was protected from overheating by Q2, $\mathrm{R} 4$ and R5. When operating at frequencies and voltages larger than $1 \mathrm{kHz}$ and $100 \mathrm{~V}$, significant heating of Q1 and Q3 can occur. They were therefore attached to heat-sinks with thermal resistance of less than $10 \mathrm{~K} / \mathrm{W}$. In addition, special care was put on designing a printed-circuit-board that minimizes the picked-up noise from the metallic-cage covered power supply. The low and high-tension paths were drawn further apart, though trying to minimize their length, and a central ground was used.

The appearance of instabilities when using amplifier circuits to drive highly-capacitive loads is quite common, mainly due to the reaction of the load to the output impedance of the circuit. We have therefore compensated the gain of the Texas Instruments TL081 with C5, added C10 and C11 to the feedback path, and included the output resistances R10 and R11. By properly choosing the values of these five components (see Fig. 1) we have obtained an amplifier that is stable for loads of up to $30 \mathrm{nF}$, a typical load value for piezoelectric motors. However, this gain in stability entails a reduction on bandwidth and slew-rate. 


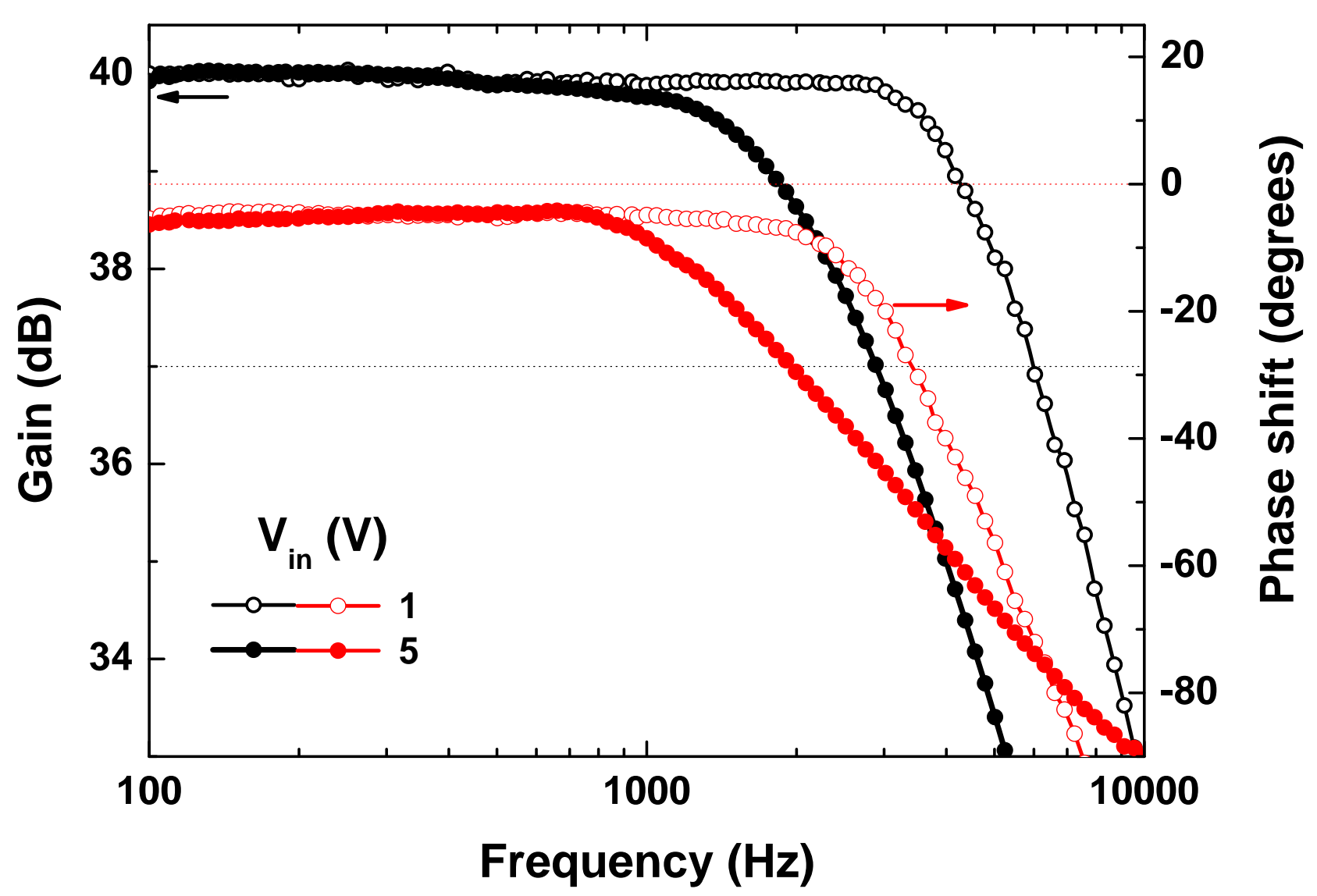

Figure 2: Gain (black points) and phase (red points) of the high-voltage amplifier loaded with $30 \mathrm{nF}$ for input signals $V_{\text {in }}$ (open symbols) and $5 \mathrm{~V}$ (full symbols). The upper red-dotted-line shows the zero-degree phase whereas the lower black one indicates the $-3 \mathrm{~dB}$ criteria for the gain.

\section{FIGURES OF MERIT OF THE AMPLIFIER}

The linearity and stability of the amplifier was tested with resistive and capacitive impedances but the performance discussed in what follows was measured using a $30 \mathrm{nF}$ load mimicking that of a piezoelectric motor. Figure 2 presents data on the gain and phase-shift of the amplifier for input signals $V_{\text {in }}=1$ and $5 \mathrm{~V}$. The $-3 \mathrm{~dB}$ criterium for bandwidth is reached at $6 \mathrm{kHz}$ for the low-input signal and decreases to $3 \mathrm{kHz}$ for the higher input. Nevertheless, Fig. 2 indicates that this amplifier is suitable to drive piezoelectric motors up to $600 \mathrm{~V}$ in the $4 \mathrm{kHz}$ range. In the case of $V_{\text {in }}=1 \mathrm{~V}$ the phase-shift of the amplifier decreases faster than the gain, being much smaller than -45 degrees at the $-3 \mathrm{~dB}$ criterium for the gain. For high input signals the phase is roughly -45 degrees at the $-3 \mathrm{~dB}$ criterium as shown for the $V_{\text {in }}=5 \mathrm{~V}$ case of Fig. 2.

\begin{tabular}{|c|c|}
\hline Voltage gain $(1 \mathrm{kHz})$ & $(100 \pm 5)$ \\
\hline Input power & $650 \mathrm{~V} \mathrm{DC}, 0.1 \mathrm{~A}$ \\
\hline Input signal & $0-10 \mathrm{~V}$ \\
\hline Input impedance & $1 \mathrm{k} \Omega$ \\
\hline Output voltage & $0-600 \mathrm{~V}$ \\
\hline Output current & $0.1 \mathrm{~A} \mathrm{rms}$ \\
\hline Power output & $60 \mathrm{~W}$ \\
\hline
\end{tabular}

Table I: Technical data of the amplifier loaded with a $30 \mathrm{nF}$ capacitor. 


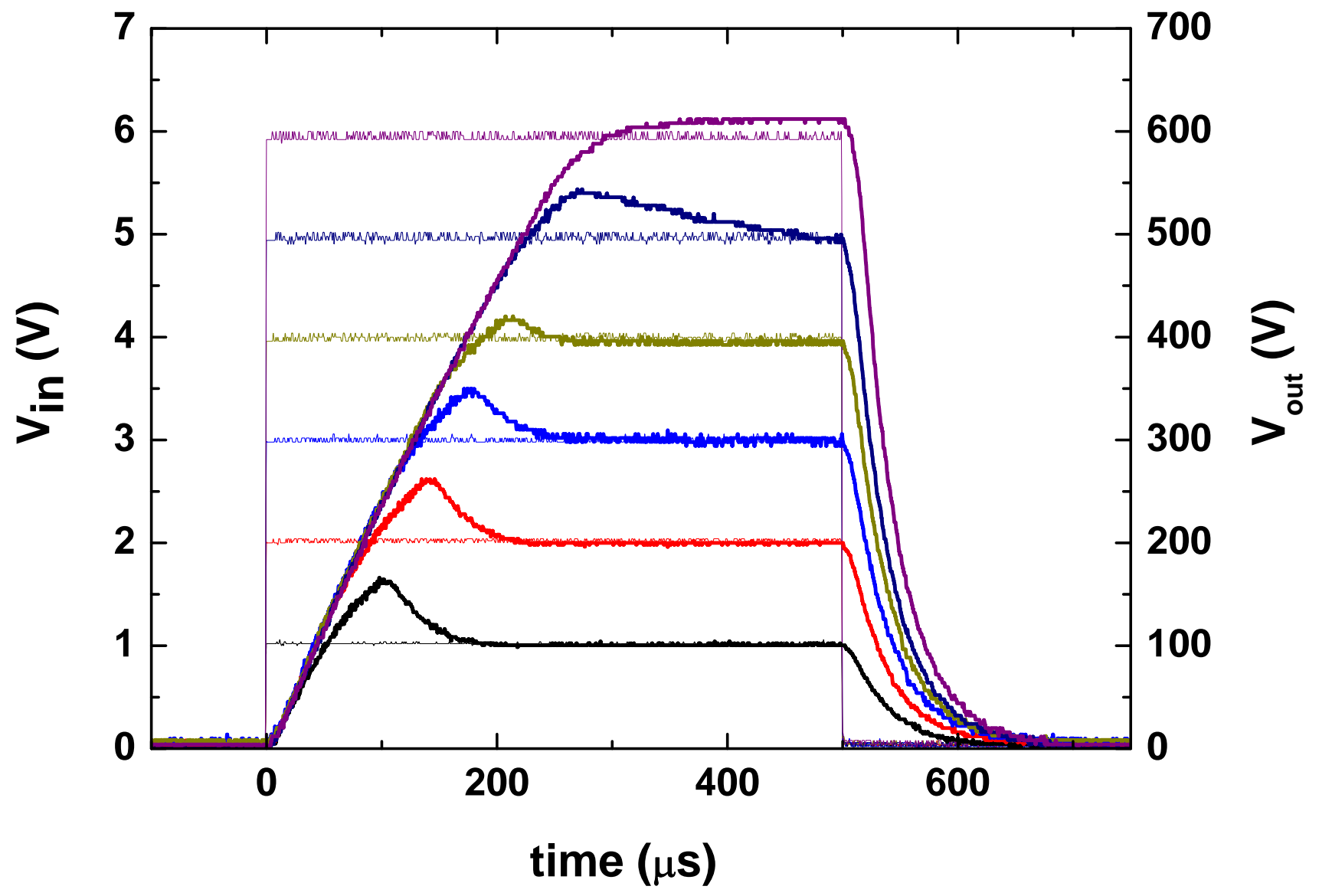

Figure 3: Response of the high-voltage amplifier, $V_{\text {out }}$, to square pulses $V_{\text {in }}$ of $500 \mu$ s and amplitudes ranging 1 to $6 \mathrm{~V}$. The amplifier is loaded with a $30 \mathrm{nF}$ capacitance.

Figure 3 shows the response of the amplifier to a square pulse of $500 \mu \mathrm{s}$ with input signals amplitude ranging from 1 to $6 \mathrm{~V}$. On increasing $V_{\text {in }}$ within this range the rise time enhances between $(80 \pm 3)$ to $(213 \pm 3) \mu$ s; on the contrary, the fall time does not evolve too much varying between $(80 \pm 3)$ and $(77 \pm 3) \mu \mathrm{s}$. The settling time increases between $(190 \pm 2)$ and $(500 \pm 2) \mu \mathrm{s}$ for $V_{\text {in }}$ between 1 and $5 \mathrm{~V}$. For the larger input signal of $6 \mathrm{~V}$ the settling time is larger than $500 \mu \mathrm{s}$. Overshooting decreases on increasing the $V_{\text {in }}$ amplitude from 50 to $8 \%$ between 1 and $5 \mathrm{~V}$. The slew-rate changes between 2.1 and $2.3 \mathrm{~V} / \mu$ s on increasing the input signal. This slew-rate for a piezoelectric load of $30 \mathrm{nF}$ is given by the $100 \mathrm{~mA}$ output current limit of the amplifier circuit plus the limiting resistances R10 and R11.

The finite slew-rate of the amplifier has an important effect on the performance of the system to follow the sawtooth signals typically used to drive piezoelectric motors. Figures 4 (a) to (d) show the output signal for pure sawtooth input waveforms with the same high-input peak voltage of $6 \mathrm{~V}$ in the range of 2 to $5 \mathrm{kHz}$. Up to $4 \mathrm{kHz}$ the amplifier reasonably follows the input signal with falling times between 40 and $70 \mu$ s and a maximum signal decrease of up to $40 \%$ with respect to the theoretical value (100 times the input). For the $5 \mathrm{kHz}$ waveform the output signal is attenuated roughly $60 \%$ and the amplifier starts to worsen its performance for larger frequencies. This is due to the finite bandwidth of the amplifier that produces an appreciable gain reduction (see Fig. 2). In addition, the amplifier slew-rate affects the output signal producing a larger deviation from the theoretical value for the minimum rather than for the maximum input signals. Figures 4 (e) and (f) show the performance of the amplifier to follow sawtooth signals with increasing and decreasing slopes of 90 and $10 \%$, respectively, and input signals with the same frequency of $4 \mathrm{kHz}$, and peak voltages of 4 and $5 \mathrm{~V}$. This figure shows that the amplifier presented here can be reliably used to drive piezoelectric motors with sawtooth signals of up to $4 \mathrm{kHz}$ and high-voltages between 400 and $600 \mathrm{~V}$.

Figure 5 (a) shows the typical output drift over a $400 \mathrm{~ms}$ time-window. In the absence of input signal the mean peak-to-peak drift is below $10 \mu \mathrm{V}$. The amplitude of the drift signal increases roughly two orders of magnitude for $V_{\text {in }}=1 \mathrm{~V}$. The output noise level in a large frequency range up to $100 \mathrm{kHz}$ is below $2 \mu \mathrm{V} / \sqrt{\mathrm{Hz}}$, see Fig. 5 (b). The spectrum presents two appreciable peaks at 75 and $250 \mathrm{~Hz}$, well below the typical operation frequencies. The $1 / f$ noise 
dominates the frequency range above $700 \mathrm{~Hz}$ and no significant features appear in the 1 to $5 \mathrm{kHz}$ range in which the amplifier is meant to be applied.

Finally, we stress that this amplifier was successfully applied to drive a coarse-approach piezoelectric motor of a home-built scanning tunnelling microscope head of the Pan-design type [10]. The amplifier was used to reliably drive this motor with sawtooth signals up to $600 \mathrm{~V}$ and $5 \mathrm{kHz}$.

\section{ADVANTAGES OF OUR HIGH-VOLTAGE AMPLIFIER TO DRIVE SLIP-STICK MOTORS}

Several works on high-voltage amplifiers applied to drive piezoelectric motors are available in the literature. In what follows we survey these works in order to highlight the advantages of our design to particularly, though not exclusively, drive coarse-approach piezoelectric motors. We start by considering a previously reported bi-polar dc-coupled low-cost high-voltage amplifier based on discrete MOSFET components with a topology similar to that of our amplifier [14]. Since low-voltage components are used, this amplifier provides $\pm 200 \mathrm{~V}$. It is useful up to $100 \mathrm{kHz}$, and maximum current and slew-rate of $340 \mathrm{~mA}$ and $300 \mathrm{~V} / \mu$ s are claimed. However, it is worth mentioning that these performances were obtained with a resistive load and no quantitative information on the reduction of the same magnitudes when loading it with some $\mathrm{nF}$ capacitors is provided.

Another bipolar amplifier with similar configuration to our design was previously proposed [15] providing $\pm 800 \mathrm{~V}$ but no much data on its figures of merit is provided besides a claim on a $100 \mathrm{kHz}$ bandwidth for $2 \mathrm{nF}$ loads. Data on the response of the amplifier to a $330 \mathrm{~Hz}$ sawtooth signal for this load allow us to estimate a relatively large fall-time of $500 \mu \mathrm{s}$ for $300 \mathrm{~V}$ output. Additionally, one main drawback of this amplifier is that presents an important overshoot.

Three other works [16-18] propose amplifier circuits based on discrete MOSFET technology providing maximum voltages between $\pm 500 \mathrm{~V}$ but all of them are bipolar. As mentioned, single-polarity amplifiers are best suited to drive coarse approach piezoelectric motors. In addition, the amplifiers presented in these three works are not particularly suited to drive piezoelectric motors with loads in the 10 to $30 \mathrm{nF}$ range since they provide output currents of $1.9,10$ and $20 \mathrm{~mA}$ as reported in Refs. 16-18. Another proposal of a MOSFET-technology-based amplifier solves this problem by presenting a dual amplifier with improved 1.9 A output current [13]. However this circuit provides an output of only $\pm 30 \mathrm{~V}$ since it is meant for megahertz piezoelectric actuators with typical loads of hundreds of $\mathrm{F}$.

Two other different approaches for this application are worth mentioning. The first of them presents five bipolar high-voltage amplifiers using the more discrete configuration provided by bipolar transistors [19]. Two of them provide $\pm 550 \mathrm{~V}$ with high output currents $(80$ and $800 \mathrm{~mA}$ ) and $\mathrm{MHz}$ bandwidth but these performances are achieved for capacitive loads between two or three orders of magnitude smaller than tens of $\mathrm{nF}$. The second approach is based on the opposite concept of a lower level of discreteness by using commercial high-voltage amplifiers from Apex Microtechnology [20]. This work introduces an auxiliary-capacitor technique in order to speed up the charge and discharge of the piezoelectric motor and consequently improve its performance.

The high-voltage amplifier introduced here has several comparative advantages with the discussed previous circuits to drive coarse-approach piezoelectric motors with loads in the $\mathrm{nF}$ range, particularly in terms of its single-polarity, maximum output of $600 \mathrm{~V}, 100 \mathrm{~mA}$ output current, and state-of-the-art noise level. In addition, the amplifier is based in MOSFET technology components available in any local market. Therefore this design is an easy-accessible solution to the problem of providing sufficiently high transient currents and output voltages to drive piezoelectric coarse-approach motors for scanning probe microscopes.

\section{ACKNOWLEDGEMENTS}

We acknowledge Pablo Pedrazzini for a careful reading of the manuscript, and Miguel Sofo Haro and Horacio Arnaldi for technical assistance. This research was partly supported by the PICT program of the Agencia Nacional de Promoción Científica under grants numbers 2007-00890 and PRH 2008-294. C.B. holds an Instituto Balseiro-CNEA scholarship and M. G. B. and Y. F. are permanent scientific researchers from Conicet.

${ }^{a}$ Author to whom correspondence should be addressed. Electronic mail: Yanina.Fasano@cab.cnea.gov.ar.

[1] G. Binnig and H. Rohrer, Helv. Phys. Acta 55, 726 (1982).

[2] G. Binnig, C. F. Quate, and C. Gerber, Phys. Rev. Lett. 56, 930 (1986).

[3] Y. Martin, and K. Wickramasinghe, Appl. Phys. Lett. 50, 20 (1987).

[4] D.W. Pohl, W. Denk, and M. Lanz, Appl. Phys. Lett. 44, 651 (1984). 
[5] N. Shimizu, T. Kimura, T. Nakamura, and I.Umebu, J. Vac. Sci. Technol. A 8, 333 (1990).

[6] D. W. Pohl, Rev. Sci. Instrum. 58, 54 (1987).

[7] Ph. Niedermann, R. Emch, and P. Descouts, Rev. Sci. Instrum. 59, 368 (1988).

[8] J. W. Lyding, S. Skala, J. S. Hubacek, R. Brockenbrough, and G. Gammie, Rev. Sci. Instrum. 59, 1897 (1988).

[9] Ch. Renner, Ph. Niedermann, A. D. Kent, and Ø. Fischer, Rev. Sci. Instrum. 61, 965 (1990).

[10] S. H. Pan, E. W. Hudson, and J. C. Davis, Rev. Sci. Instrum. 70, 1459 (1999).

[11] Nanonis, Scanning Probe Microscopy Control System.

[12] RHK Technology, Inc., Scanning Probe Microscopy Control Systems.

[13] A. J. Fleming, Rev. Sci. Instrum. 80, 104701 (2009).

[14] M. S. Colclough, Rev. Sci. Instrum. 71, 4323 (2000).

[15] L. M. Eng, F. Eng, Ch. Seuret, A. Kündig, and P. Günter, Rev. Sci. Instrum. 67, 401 (1996).

[16] C. Barchesi, R. Generosi, and A. Cricenti, Rev. Sci. Instrum. 64, 3521 (1993).

[17] V. Ström, R. D. Gomez, J. Nogués, B. Rodell, and K. V. Rao, Meas. Sci. Technol. 6, 1072 (1995).

[18] D. V. Pelekhov, J. B. Becker, and G. Nunes Jr., Rev. Sci. Instrum. 70, 114 (1999).

[19] H. Müller, Rev. Sci. Instrum. 76, 084701 (2005).

[20] K. Chatterjee, M. C. Boyer, W. D. Wise, and E. W. Hudson, Rev. Sci. Instrum. 80, 095110 (2009). 

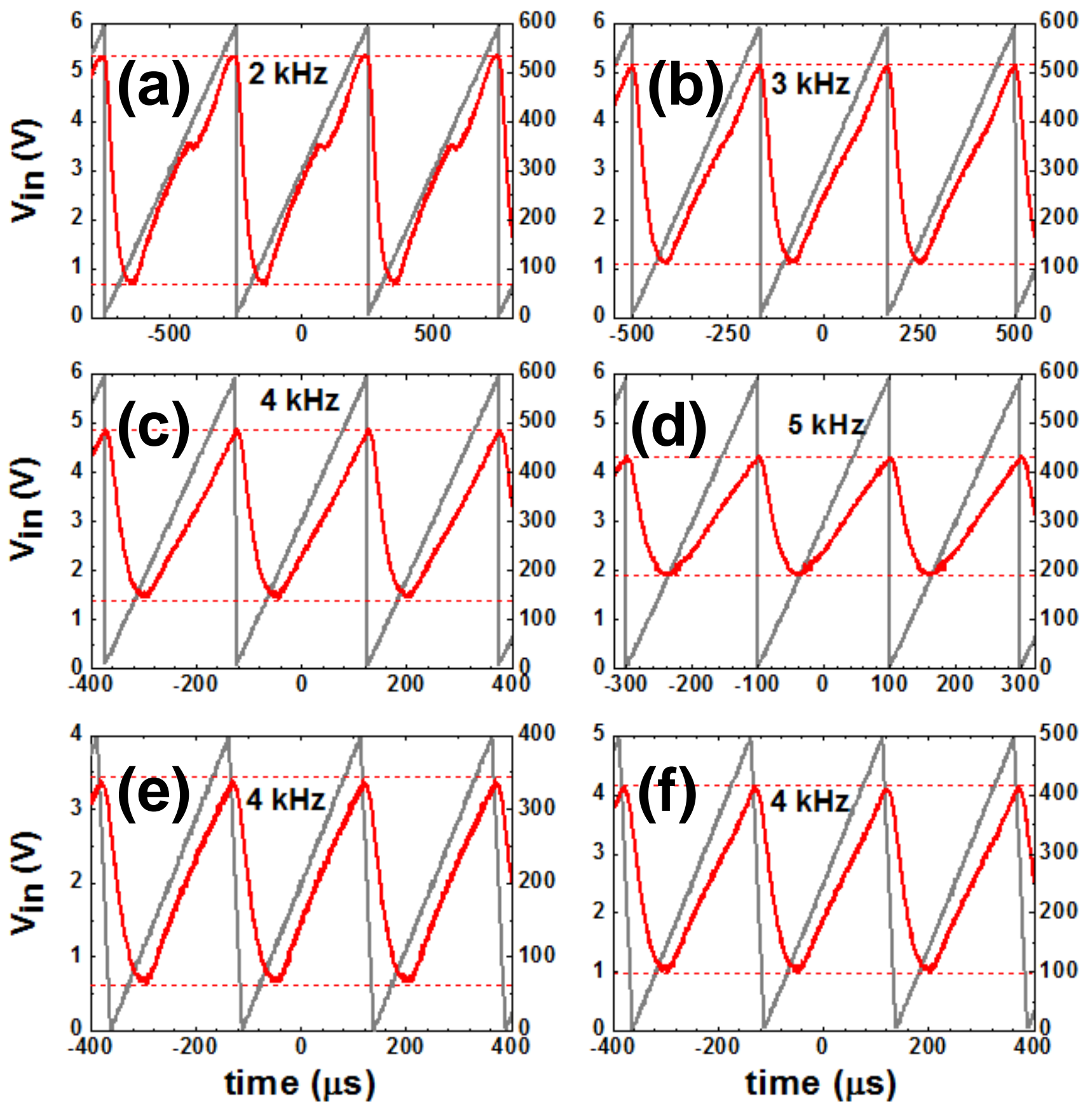

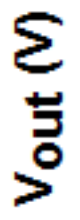

Figure 4: Output signals (red curves) for sawtooth waveform input signals (gray curves).(a), (b), (c), and (d) Response to pure sawtooth signals with the same peak voltage of $6 \mathrm{~V}$ and frequencies ranging from 2 to $5 \mathrm{kHz}$. (e) and (f)Response to 90-10\% slopes sawtooth waveform input signals with the same frequency of $4 \mathrm{kHz}$ and peak voltages of 4 and $5 \mathrm{~V}$. Red-dotted lines indicate the minimum and maximum attenuated values of the output signal. 

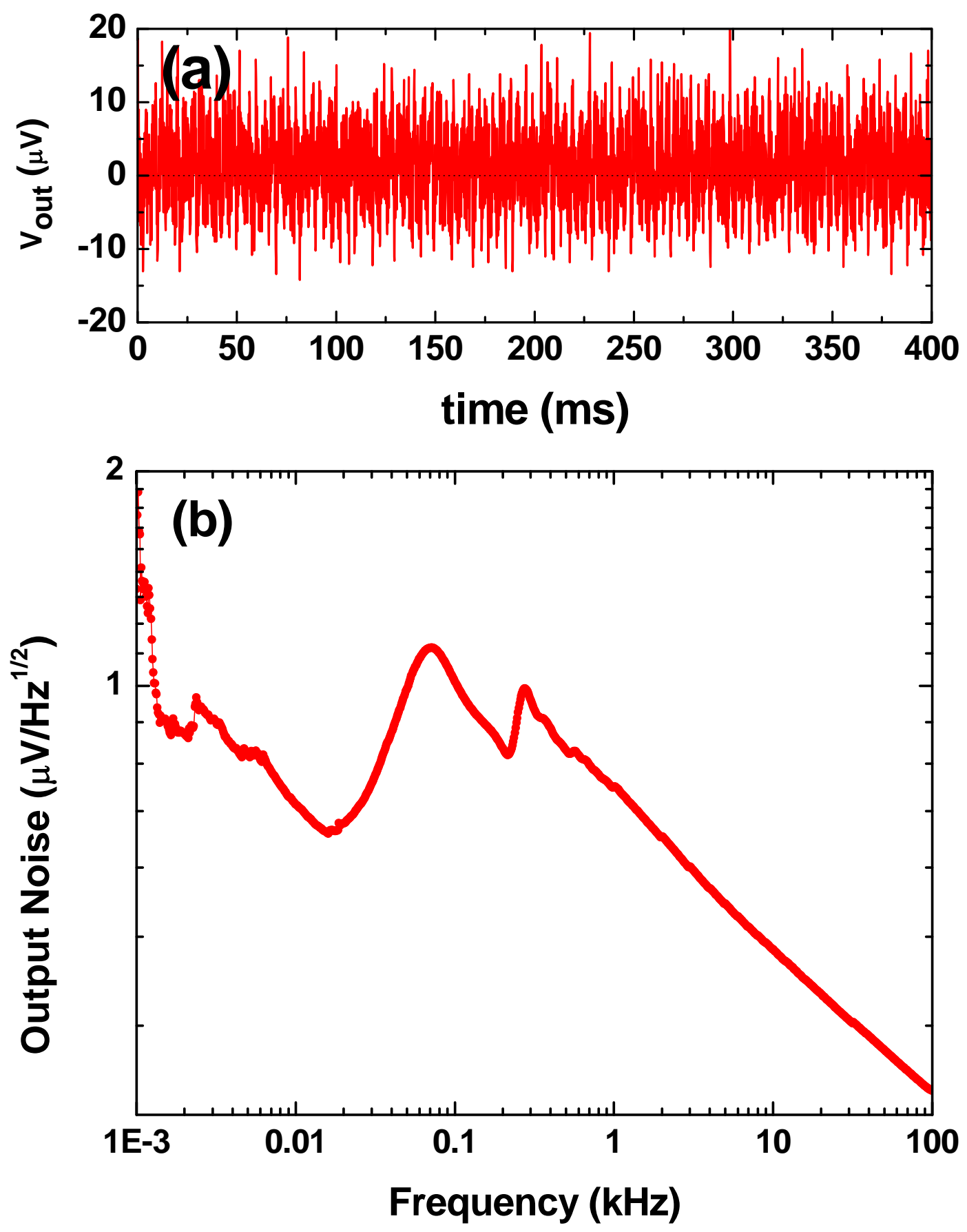

Figure 5: Noise in the amplifier output signal for zero input. (a) Drift during a $400 \mathrm{~ms}$ time-window. (b) Output noise spectrum in a large frequency window. All data were acquired by means of a lock-in amplifier. 